\title{
An Approach For Obstacle Detection System For Vehicle Using Iot
}

\author{
Mr. G. Kalyan Chakravarthi ${ }^{1}$, K. Sai Vinayak ${ }^{2}$, Fazal Abbas ${ }^{3}$, P.B. PurnaSri ${ }^{4}$, T. Akhil ${ }^{5}$, \\ Department of Computer Science and Engineering ${ }^{1,2,3,4,5}$, Gayatri Vidya Parishad College for Degree and PG Courses ${ }^{1,2,3,4,5}$, \\ Email: gkalyanchakravarthi@gvpcdpgc.edu.in ${ }^{1}$, saivinayakkandregula@gmail.com²,fazalabbas143@gmail.com ${ }^{3}$, \\ purnasrijuly18@gmail.com ${ }^{4}$,tarivitlaakhil@gmail.com ${ }^{5}$
}

\begin{abstract}
Vehicles are the mode of transportation in our day to day life. Considering four wheelers, six and many more heavy vehicles, it is quite a difficult job for the driver to have a glance on the four sides of the vehicle, which is one of the cause for road accidents that leads to major damage to the vehicles and even severe injuries to the occupants of the vehicles. When the vehicle is at rest position there were many chances for the pets to go under the vehicle and also some obstacles like rocks may exist under the vehicle. When the driver starts the vehicle he/she will never have a look under the vehicle which may cause damage to the vehicle and nudging of pets which may lead to their death. This project is designed to overcome the above problems Raspberry Pi and Ultrasonic sensors are used to monitor the presence of obstacles around and under the vehicle. The distance monitored is displayed on user interface. An alert is provided to the driver when the obstacle or any other vehicles are nearer to the vehicle. This system also monitors the obstacles under the vehicle and shows a message on the user interface regarding the presence of an obstacle under the vehicle. This helps the driver to drive accordingly and to have control over the vehicle which assures a safe drive.
\end{abstract}

Keywords- Obstacle, Raspberry Pi, Ultrasonic Sensor, Safe drive.

\section{INTRODUCTION}

Road accidents are most common issues in our daily lives. These accidents lead to major life loss and even severe vehicle damage. The automobile industries have come up with different aspects to overcome these accidents. Cars are installed with radar sensors and camera on rear side to monitor the obstacles. But using radar sensors on rear side of the vehicle doesn't solve this problem.

So in this project sensors are used on four sides and under the vehicle to detect the obstacles [3]. This provides a safe drive to the driver and life of the pets can be saved. It is more efficient and economically cheap as cameras are not used. Instead ultrasonic sensors are used to monitor obstacle presence and distance to them.

\section{EXISTING SYSTEM}

\section{- $\quad$ Automated Cars}

Many automobile companies are launching and about to launch the automated cars in which the car works on self-mode by detecting the obstacles and taking necessary actions. Everything will be under the control of car itself.

- Camera and Radar sensors on rear side of the cars

Now-a-days cars are installed with camera and radar sensors on rear side of the vehicle and provides user an alert when there is an obstacle near to the vehicle. The video is displayed on the screen in the car. By having a glance on that screen, driver drives the car accordingly. But in these both cases it is not a cost efficient methods.

\section{PROPOSED SYSTEM}

This section describes the orientation and working of the proposed system named 'IoT BASED OBSTACLE DETECTION SYSTEM FOR VEHICLES'. The software and hardware specifications required to govern this functionality is described in this section.

\subsection{Software specifications}

This section deals with the modules that receive data from the sensors. The result is calculated and sent back to the hardware unit to warn the driver. The modules involved in this proposed system are:

- Obstacle detection module

- Distance calculation module

\subsection{Hardware specifications}

The proposed system designed with Raspberry Pi which is a series of small single board computer that is used to process the data received from sensors. The hardware components used in the design of the proposed system are:

- Raspberry Pi 


\section{Available online at www.ijrat.org}

- Ultrasonic Sensor

- Buzzer

- Breadboard and jumper wires.

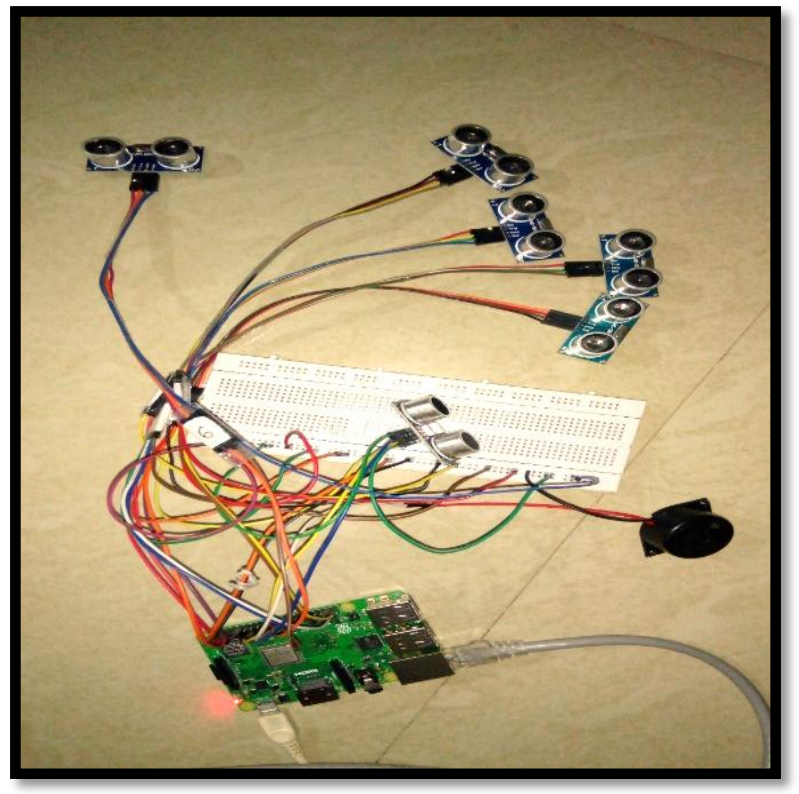

Fig. 1. Connectivity of obstacle detection system for vehicles

\section{PRINCIPLE AND METHODOLOGY}

The principle involved in this project is to measure the distance between the vehicles and obstacles with the help of ultrasonic sensors. When an obstacle is detected around the vehicle the distance is measured and sent to the user interface to display those measurements [1]. And when the obstacle is detected under the vehicle the data is sent to user interface and displays a message whether obstacle is detected or no obstacle is detected. The distance is calculated by using the formula:

$$
\text { Distance }=\frac{\text { speed of sound } * \text { time taken }}{2}
$$

The methodology involved in this system is to transmit and receive the ultrasonic signals [2]. With the help of these signals the time and speed is calculated. By using these the distance is calculated. When the distance at any sensor is less than or equal to $30 \mathrm{~cm}$ then buzzer is triggered as an alarm to the driver.

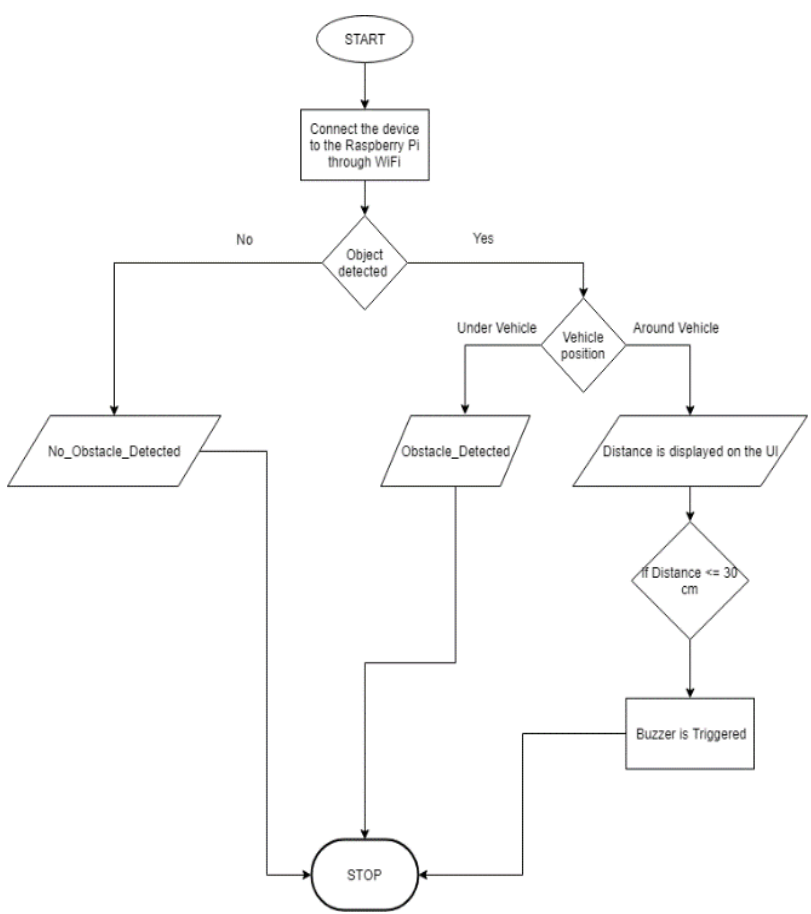

Fig. 2. Flow chart of the system

\section{EXPERIMENTAL RESULTS AND ANALYSIS}

Experiments are conducted to detect the distance between vehicles and obstacles. As shown in the table obstacles are placed very nearer to the vehicle i.e., less than $30 \mathrm{~cm}$ on all four sides and as a result, the buzzer is triggered. An obstacle is placed under the vehicle and checked whether the user interface is displaying 'Obstacle_Detected' or not. In another case i.e., no obstacle placed under the vehicle, the user interface is checked whether it is displaying 'No_Obstacle_Detected' or not. These experimental results are shown in tables 1,2 . The images related to these experiments are shown in figures 3,4,5 respectively.

Table 1. Experimental setup for obstacle distance detection and its observed result.

\begin{tabular}{|c|c|c|}
\hline \multicolumn{3}{|c|}{ Obstacle Distance Detection } \\
\hline Obstacle & $\begin{array}{c}\text { Distance between } \\
\text { vehicle and } \\
\text { obstacles }\end{array}$ & Detected(Y/N) \\
\hline Box & 20 & Yes \\
\hline Box & 50 & Yes \\
\hline Vehicle & 30 & Yes \\
\hline Vehicle & 100 & Yes \\
\hline
\end{tabular}

Table 2. Experimental setup for obstacle detection and its observed result.

\begin{tabular}{|c|c|c|}
\hline \multicolumn{3}{|c|}{ Obstacle Detection } \\
\hline Obstacle & Expected Result & Result \\
\hline
\end{tabular}


International Journal of Research in Advent Technology, Vol.7, No.4S, April 2019

E-ISSN: 2321-9637

\section{Available online at www.ijrat.org}

\begin{tabular}{|c|c|c|}
\hline placed(Y/N) & & matched(Y/N) \\
\hline Yes & Obstacle_Detected & Yes \\
\hline No & No_Obstacle_Detected & Yes \\
\hline
\end{tabular}

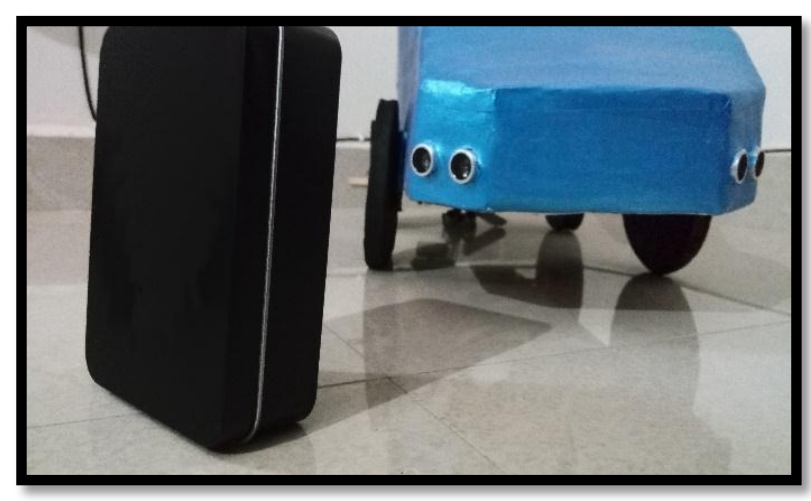

Fig. 3. Detecting obstacle at front side of the vehicle

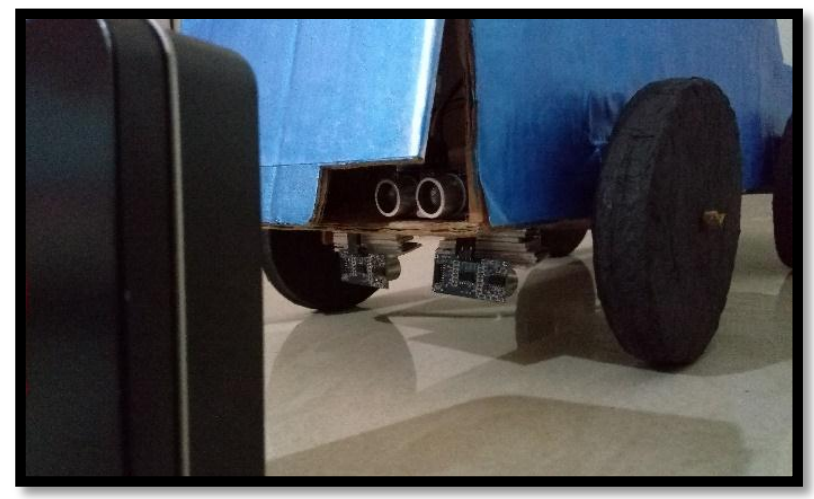

Fig. 4. Detecting obstacle at rear side of the vehicle

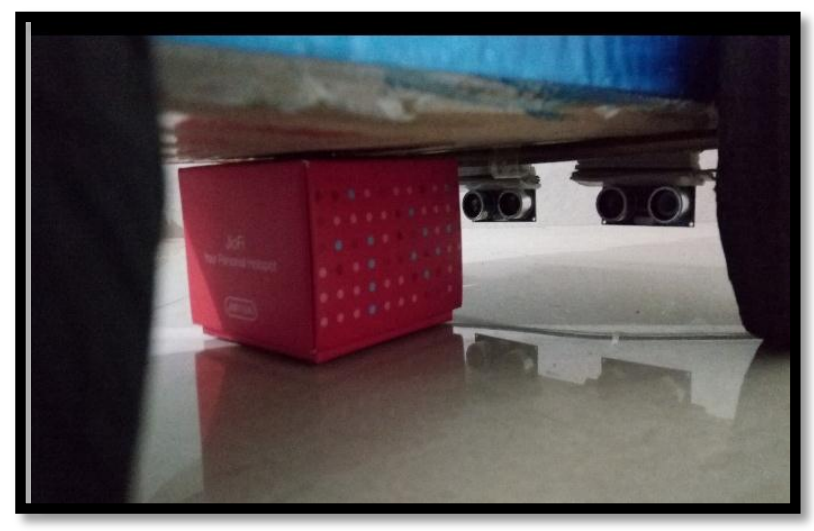

Fig. 5. Detecting obstacle under the vehicle

\section{CONCLUSION AND FUTURE WORK}

A reliable system is developed to detect the obstacles and alert the driver whenever the obstacles or vehicles are very nearer to the vehicle. It is cost-efficient and easy to use. A safe drive is assured to the driver. As a future work, it can be implemented with Bluetooth connectivity and can be installed on the car screen. A voice alert can be added when an obstacle is detected under the vehicle.

\section{REFERENCES}

[1] S. Anusha (EIE), G.S. Sandhya (CH), "A Literature Survey On Obstacle Detection And Their Movement For Automobiles", International Journal of Innovative Technology and Research,Volume-4,Issue-6, 2016.

[2] N. Harish Kumar, G. Deepak, J. Nagaraja, "An IoT based Obstacle Detection and Alerting System in Vehicles using Ultrasonic Sensor", International Journal of Engineering Research \& Technology, ISSN: 22780181, NCETEIT - 2017 Conference Proceedings.

[3] R. Surya Kumar, P. Kingston Stanley, A. Sanjeevi Gandhi, "Raspberry Pi based vehicle collision avoidance system", Institute of Electrical and Electronics Engineers. 